\title{
Study on the enrichment of Sulfur Hexafluoride in the tmosphere through polyimide hollow fiber membrane
}

\author{
Weixian Wang ${ }^{\mathrm{a}}$, Yaoqin Wang, Weiping Li, Yuan Yuan \\ Northwest Institute of Nuclear Technology, XI'AN 710024, China
}

\begin{abstract}
Sulfur hexafluoride is per molecule the strongest greenhouse gas know, the features have brought SF6 into the climatic impact discussion aimed at reduction of emissions. The separation effects of sulfur hexafluoride in the atmosphere are studied through polyimide hollow fiber membrane with different conditions on pressure drop, gas flow and temperature. The sulfur hexafluoride concentration increased with increased pressure drop of the membrane, increased temperature and decreased non-filtrate flow flux; the recovery of sulfur hexafluoride exceeds $93 \%$, enrichment coefficient was 18.5; sulfur hexafluoride is not detected at the flux of the filtrate flow, which means sulfur hexafluoride is riddled by membrane. The results showed that polyimide hollow fiber membrane can effectively separate sulfur hexafluoride from mixed gas
\end{abstract}

\section{Introduction}

The stabile trace sulfur hexafluoride in the atmospheric is produced by human activities[1]. $\mathrm{SF}_{6}$ is mainly used in electric switch equipment as a dielectric[2] and aluminum and magnesium smelting process as a greenhouse gas[3], because which is a colorless, odorless, nontoxic and nonflammable inert gas. $\mathrm{SF}_{6}$ is $\mathrm{CO}_{2}$ on $\mathrm{GWP}_{100}$ value of 24 times, and it is one of the strongest greenhouse gases, and its volume of discharged into the atmosphere is increasing at an annual rate of $7-8 \%$. Especially in aluminum and magnesium smelting process, in fact the vast majority $\mathrm{SF}_{6}$ is released in the atmosphere without being consumed. Therefore enrichment and recycling are important problems to protect environment.

Gas membrane separation is a new separation technology, and compared with conventional separation methods such as cryogenic distillation and cryogenic adsorption, it has high separation efficiency, small coverage area, low energy consumption, simple operation, easy maintenance and less investment etc..

Polyimide hollow fiber membrane is glass state. polymer, and it is a kind of chain compound of which the main component of the membrane material is aromatic polyimide. It has higher wear resistance, heat resistance and excellent insulating property, but also it does not dissolve in any organic solvent, oil and dilute acids. In the middle period of the eighties it began to be used in industry such as the separation of $\mathrm{CO}_{2} / \mathrm{CH}_{4}, \mathrm{H}_{2} / \mathrm{N}_{2}$, $\mathrm{H}_{2} / \mathrm{CH}_{4}, \mathrm{O}_{2} / \mathrm{N}_{2}, \mathrm{CO}_{2} / \mathrm{N}_{2}$ etc.[4]. However reports on the method of $\mathrm{SF}_{6}$ separation and enrichment are rare. Studying on the enrichment technique of $\mathrm{SF}_{6}$ gas of air concentration with polyimide hollow fiber membrane, and this paper inspects operation parameters on the separation performance for the enrichment of $\mathrm{SF}_{6}$ gas of high concentration to provide a reference. The results show that, polyimide hollow fiber membrane is obvious effect about separation and enrichment of $\mathrm{SF}_{6}$.

\section{Experimental}

\subsection{Equipment and Installation}

Polyimide hollow fiber membrane separator (Shanghai Jingteng Technology Company), diameter $55 \mathrm{~mm}$, length $234 \mathrm{~mm}$. Agilent6890A gas chromatograph(equipped with a micro-cell electron capture detector $(\mu$-ECD)), the detection limit of SF6 is $2 \times 10-13 \mathrm{~L} / \mathrm{L}$. CYG1102T pressure transmitter (Xi'an Sensor Control Technology Co. Ltd.), the measurement range $0 \sim 1 \mathrm{MPa}$, precision 0.25. LZB-6 Glass rotameter(Yuyao City Yinghuan flow meters Co. Ltd.), the measurement range $0.06 \sim 0.6 \mathrm{~m} 3 / \mathrm{h}$, accuracy 2.5. LZB-3 Glass rotameter (Yuyao City Yinghuan flow meters Co. Ltd.), the measurement range $0.1 \sim 1 \mathrm{~L} / \mathrm{min}$, accuracy 4 . High purity nitrogen $99.9995 \%$. compressed air. Concentration of SF6 gas is $6.5 \times 10-12$ $\mathrm{L} / \mathrm{L}$. The schematic diagram of the experimental system is shown in figure 1.

\footnotetext{
a Corresponding author: wiges@sina.com
} 


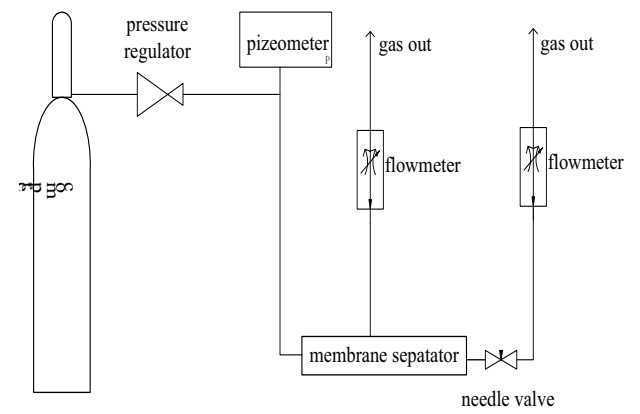

Figure 1. Schematic diagram of the experimental system

\subsection{Experimental method}

In the experiment pressure reducing valve controls differential pressure, needle valve controls impermeable gas flow, permeable gas is natural emptying. The measurement of $\mathrm{SF}_{6}$ concentration that it was separated by polyimide hollow fiber membrane was conducted using a gas chromatograph (Agilent6890A, USA).

\subsection{Separation and Enrichment performance evaluation}

In present work, we provide enrichment coefficients and recovery for Using to evaluate the result that $\mathrm{SF}_{6}$ of the air was separated and enriched by Polyimide hollow fiber membrane.

The definition of enrichment coefficient is the ratio of $\mathrm{SF}_{6}$ concentration in the air and in the impermeable gas such that:

$$
Y=\frac{V_{1}}{V_{2}}
$$

Where $\mathrm{Y}$ is enrichment coefficient, $\mathrm{V}_{1}(\mathrm{~L} / \mathrm{L})$ is $\mathrm{SF}_{6}$ concentration in the air and $\mathrm{V}_{2}(\mathrm{~L} / \mathrm{L})$ is $\mathrm{SF}_{6}$ concentration in the impermeable gas.

Recovery calculation formula such that:

$$
\mathbf{P}=\frac{F_{1} \times C_{1}}{F_{1} \times C_{1}+F_{2} \times C_{2}}
$$

Where $\mathrm{P}$ is recovery of $\mathrm{SF}_{6}, \mathrm{C}_{1}(\mathrm{~L} / \mathrm{L})$ is $\mathrm{SF}_{6}$ concentration in the impermeable gas, $\mathrm{C}_{2}(\mathrm{~L} / \mathrm{L})$ is $\mathrm{SF}_{6}$ concentration in the permeable gas, $\mathrm{F}_{1}(\mathrm{~L} / \mathrm{min})$ is the flow of the impermeable gas and $\mathrm{F}_{2}(\mathrm{~L} / \mathrm{min})$ is the flow of the permeable gas.

In the recovery formula we require the $\mathrm{SF}_{6}$ concentration in the permeable gas, but the $\mathrm{SF}_{6}$ concentration of the permeable gas was lower than the gas chromatograph detection limit in the experiment. Therefore for calculating recovery, we think that $\mathrm{SF}_{6}$ concentration in permeability gas is the detection limit about that $\mathrm{SF}_{6}$ is analysed by gas chromatograph.

\section{Result and Discussion}

3.1 Infiltration time has influence on enrichment of $\mathrm{SF}_{6}$
At this test temperature of $25^{\circ} \mathrm{C}$, through pressure reducing valve controls, that the membrane differential pressures is $0.1 \mathrm{MPa}, 0.15 \mathrm{MPa}, 0.2 \mathrm{MPa}$ and $0.25 \mathrm{MPa}$, respectively. Through needle valve controls, that the flow of impermeable gas is $0.4 \mathrm{~L} / \mathrm{min}$. In the above conditions, respectively in $0.5 \mathrm{~min}, 1.5 \mathrm{~min}, 3 \mathrm{~min}$ sampling, then every 3 min sampling.

The schematic diagram of that the $\mathrm{SF}_{6}$ concentration in the impermeable gas changes with time is shown in figure 2 .

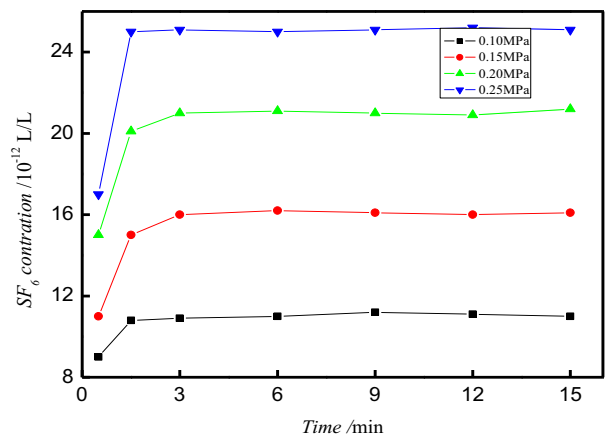

Figure 2. Relationship of $\mathrm{SF}_{6}$ concentration and infiltration time

At different pressure differentials, $\mathrm{SF}_{6}$ gas concentration is basic balance after three minutes. The $\mathrm{SF}_{6}$ concentration in the impermeable gas is to stabilize in a relatively short period, which show that the ability of adsorbing and dissolving in polyimide hollow fiber membrane separator is weak, and $\mathrm{SF}_{6}$ is separated fast from the mixed gases.

3.2 The result of differential pressure at membrane separator influencing on $\mathrm{SF}_{6}$ enrichment

Fig. 3 shows the result that differential pressure at membrane separator influences on $\mathrm{SF}_{6}$ enrichment when the temperature is $25^{\circ} \mathrm{C}$ and the flow is $0.1 \mathrm{~L} / \mathrm{min}, 0.2$ $\mathrm{L} / \mathrm{min}, 0.4 \mathrm{~L} / \mathrm{min}, 0.6 \mathrm{~L} / \mathrm{min}, 0.8 \mathrm{~L} / \mathrm{min}$ and $1.0 \mathrm{~L} / \mathrm{min}$, respectively. With increasing the pressure difference, the concentration of the gas is increase in membrane separator, therefore the concentration of $\mathrm{SF}_{6}$ in impermeable gas shows a trend of increase. When membrane separator differential pressure is certain, the concentration of $\mathrm{SF}_{6}$ shows a trend of increase with the flow of impermeable gas increasing. The above phenomenon indicates that when the flow of impermeable gas increasing, the gas flow velocity is increase and the residence time is reducing in membrane separator[5], so that the percolation of $\mathrm{O}_{2}$ and $\mathrm{N}_{2}$, etc. decreases, and the content in the permeability gas increases. 


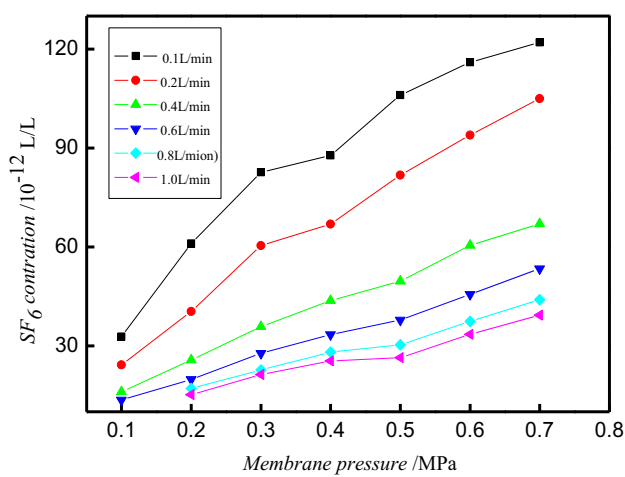

Figure 3. The effect of different pressure influence on $\mathrm{SF}_{6}$ separation

\subsection{The effect of temperature influence on $\mathrm{SF}_{6}$ separation}

When the impermeable gas flow is $0.4 \mathrm{~L} / \mathrm{min}$, and the temperature is $18{ }^{\circ} \mathrm{C}, 25{ }^{\circ} \mathrm{C}$ and $29{ }^{\circ} \mathrm{C}$, respectively, $\mathrm{SF}_{6}$ concentration in impermeable gas is measured with the condition of differential pressure. The result shows that $\mathrm{SF}_{6}$ concentration in impermeable gas increases with increasing temperature.

Polyimide belongs to rigid polymer chain, the diffusion of gas molecules occurs mainly in the molecular chain with high packing density. Because polymer chain motion increases with higher temperature, the gaps increase in the polymer chain, accordingly $\mathrm{N}_{2}, \mathrm{O}_{2}$ and other gases diffusion coefficient increase, this phenomenon is beneficial to their penetration. So that the above cause makes $\mathrm{SF}_{6}$ gas concentration increase.

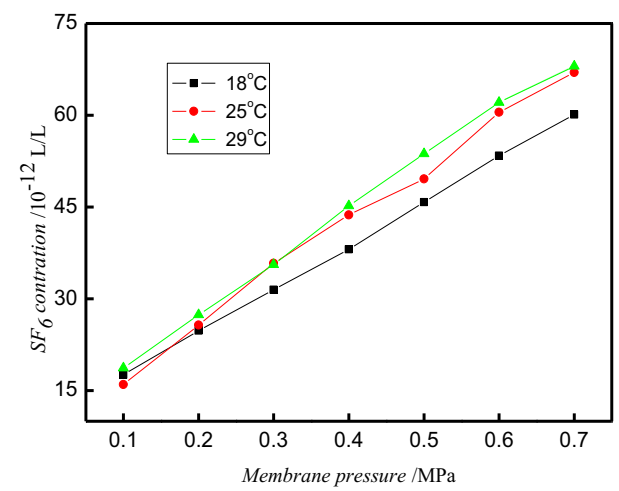

Figure 4. The effect of temperature influence on $\mathrm{SF}_{6}$ separation

3.4 The enrichment of $\mathrm{SF}_{6}$ in the atmosphere through polyimide hollow fiber membrane

\subsubsection{Enrichment coefficient}

According to the experimental results, $\mathrm{SF}_{6}$ gas concentration in the atmosphere is $6.5 \times 10^{-12} \mathrm{~L} / \mathrm{L}$ and the largest concentration of the $\mathrm{SF}_{6}$ enrichment is $1.2 \times 10^{-10}$ $\mathrm{L} / \mathrm{L}$. Through calculating $\mathrm{SF}_{6}$ enrichment coefficient is 18.5 .

According to the penetration amount formula :

$$
\mathrm{Q}=\mathrm{S} \times \mathrm{A}-\triangle \mathrm{P} / \mathrm{L}
$$

Where $\mathrm{Q}$ is the gas penetration amount, $\mathrm{A}$ is membrane area, $\mathrm{L}$ is membrane thickness, $\triangle \mathrm{P}$ is the differential pressure about tow ends and $\mathrm{S}$ is the gas penetration coefficient.

According to the formula analysis, increasing the membrane area can increase the gas penetration amount. Therefore increasing the $\mathrm{SF}_{6}$ concentration of the impermeable gas or by multiple membrane separation, all which can make $\mathrm{SF}_{6}$ enrichment coefficient increase.

\subsubsection{Recovery rate}

Fig.5 shows the result that the different recovery rate of $\mathrm{SF}_{6}$ at different condition. The recovery rate of $\mathrm{SF}_{6}$ is up to $93 \%$ with polyimide hollow fiber membrane separator enrichment, which mainly reason is polyimide belongs to rigid polymer chain, that the rigid molecular network more like "molecular sieve", so that the different of gas molecule size decides its permeability difference with the same kind of polyimide membrane. Because $\mathrm{SF}_{6}$ molecule size is bigger, it is not easy through the aperture of smaller "molecular sieve". Based on the above reason, the $\mathrm{SF}_{6}$ concentration of the permeable gas is lower than the gas chromatograph detection limit in the experiment that is explained.

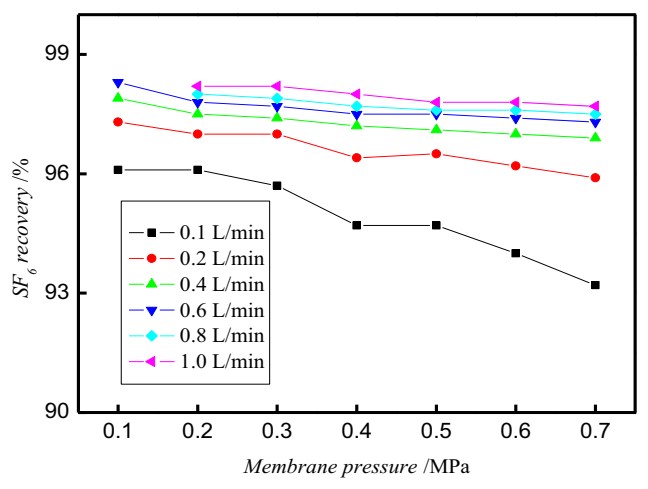

Figure 5. The recovery rate of $\mathrm{SF}_{6}$ at different condition

Under the certain flux, the membrane differential pressure increase loads to the recovery rate of $\mathrm{SF}_{6}$ reduce. Because membrane differential pressure increases, to largen the driving force, that the result leads to the flux of the penetration gas also increase. The reason changes the components of the permeable gas and causes the recovery rate of $\mathrm{SF}_{6}$ reducing.

The enrichment effect shows when the certain flux, differential pressure increases, enrichment coefficient increases and recovery rate decreases. Enrichment ratio will be considered in real application, therefore, it is main to select bigger operating pressure. Thought bigger operating pressure and lower flux, the methods all can increase the enrichment coefficient.

\section{Conclusion}

Polyimide hollow fiber membrane for separation and enrichment $\mathrm{SF}_{6}$ of in the mixed gases has good effect. 
(1) The $\mathrm{SF}_{6}$ enrichment coefficient increases with higher differential pressure and temperature and lower flux in the impermeable gas.

(2) $\mathrm{SF}_{6}$ gas recovery rate is higher to be up to $93 \%$ in the experiment

(3) The enrichment coefficient is increase with increasing differential pressure and decreasing flow, but meanwhile $\mathrm{SF}_{6}$ gas recovery rate become lower.

\section{References}

1. J. Harnisch, R. Borchers, P. Fabian, H.W. Gaeggeler, U. Schotterer, Nature[J]. 1996, 384, 32.

2. M. K. W. Ko, N. D. Sze, W. Wang, G. Shia, A. Goldman, F. Murcray, D.Murc-ray, C. Rinsland, J. Geophy[J]. Res. 1993, 98 (D6), 10499-10507.

3. Maiss, M., Brenninkmeijer, A.M. Atomspheric $\mathrm{SF}_{6}$ : Trend, source, and prospects[J]. Environ.Sci. Technol. 1998, 32, 3077-3086.

4. Ik-Jae Chung, Kwang-Rae Lee, Sun-Tak Hwang. Separation of CFC-12 from air by polyimide hollowfiber membrane module[J]. Journal of Membrane Science. 1995, 105, 177-185.

5. Muslikhin Hidayat, Anders Rasmuson. Some aspects on gas-solid flow in a U-bend: Numerical investigation. Powder Technology. 2005, 153, 1-12. 